\title{
RSC-dependent constructive and destructive interference between opposing arrays of phased nucleosomes in yeast
}

\author{
Dwaipayan Ganguli, ${ }^{1}$ Răzvan V. Chereji, ${ }^{1}$ James R. Iben, Hope A. Cole, and David J. Clark \\ Program in Genomics of Differentiation, Eunice Kennedy Shriver National Institute for Child Health and Human Development, \\ National Institutes of Health, Bethesda, Maryland 20892, USA
}

\begin{abstract}
RSC and SWI/SNF are related ATP-dependent chromatin remodeling machines that move nucleosomes, regulating access to DNA. We addressed their roles in nucleosome phasing relative to transcription start sites in yeast. SWI/SNF has no effect on phasing at the global level. In contrast, RSC depletion results in global nucleosome repositioning: Both upstream and downstream nucleosomal arrays shift toward the nucleosome-depleted region (NDR), with no change in spacing, resulting in a narrower and partly filled NDR. The global picture of RSC-depleted chromatin represents the average of a range of chromatin structures, with most genes showing a shift of the +1 or the -1 nucleosome into the NDR. Using RSC ChIP data reported by others, we show that RSC occupancy is highest on the coding regions of heavily transcribed genes, though not at their NDRs. We propose that RSC has a role in restoring chromatin structure after transcription. Analysis of gene pairs in different orientations demonstrates that phasing patterns reflect competition between phasing signals emanating from neighboring NDRs. These signals may be in phase, resulting in constructive interference and a regular array, or out of phase, resulting in destructive interference and fuzzy positioning. We propose a modified barrier model, in which a stable complex located at the NDR acts as a bidirectional phasing barrier. In RSC-depleted cells, this barrier has a smaller footprint, resulting in narrower NDRs. Thus, RSC plays a critical role in organizing yeast chromatin.
\end{abstract}

[Supplemental material is available for this article.]

The basic subunit of chromatin structure, the nucleosome, has inhibitory effects on transcription, DNA replication, and repair, which are accounted for by its compact structure and innate stability. The nucleosome core is composed of two molecules from each of the four core histones, $\mathrm{H} 2 \mathrm{~A}, \mathrm{H} 2 \mathrm{~B}, \mathrm{H} 3$, and $\mathrm{H} 4$, forming a histone octamer, around which is wrapped $\sim 147$ bp of DNA (Luger et al. 1997). Early nuclease digestion studies of chromatin in nuclei revealed a characteristic ladder of DNA bands in a gel, indicating that nucleosomes are regularly spaced along the DNA in vivo. The nucleosomes in the cells of higher eukaryotes are generally spaced farther apart ( $\sim 195$ bp per nucleosome) than in yeast ( 165 bp) (van Holde 1988). Authentic nucleosomes can be reconstituted in vitro using purified histones and DNA, but they are not regularly spaced. Regular spacing requires the addition of ATP-dependent chromatin remodeling complexes, such as ISW1, ISW2, or ACF (Ito et al. 1997; Tsukiyama et al. 1999) and is affected by linker histone (Blank and Becker 1995). The remodeling complexes use the free energy of ATP hydrolysis to move nucleosomes along DNA (Clapier and Cairns 2009; Hota and Bartholomew 2011; Narlikar et al. 2013).

Genome-wide studies of nucleosomal DNA isolated from cells by digestion with micrococcal nuclease (MNase) and analyzed using tiling microarrays or massively parallel sequencing have revealed that most yeast genes have a nucleosome-depleted region (NDR) at their promoters (Lee et al. 2004; Yuan et al. 2005). Furthermore, nucleosomes are phased with respect to the transcription start site (TSS) (Yuan et al. 2005; Lee et al. 2007; Mavrich et al. 2008). Thus, on a typical yeast gene, nucleosomes are formed into

\footnotetext{
1These authors contributed equally to this work.

Corresponding author: clarkda@mail.nih.gov

Article published online before print. Article, supplemental material, and publication date are at http://www.genome.org/cgi/doi/10.1101/gr.177014.114.
}

a regularly spaced array and phased such that the TSS is located just inside the first $(+1)$ nucleosome, and the promoter is depleted of nucleosomes.

Currently, there is great interest in deciphering the mechanisms by which nucleosome phasing occurs in vivo. The most satisfying explanation for phasing is supplied by the barrier model, which posits that promoter-bound proteins [or possibly specific DNA sequences, such as poly(dA-dT)] act as a steric barrier to nucleosome assembly. Thus, nucleosomes form on the DNA adjacent to the barrier, resulting in phasing relative to the barrier (Kornberg 1981; Mavrich et al. 2008; Chereji and Morozov 2011; Chereji et al. 2011; Rube and Song 2014). ATP-dependent chromatin remodelers then assemble a correctly spaced nucleosomal array, beginning with the nucleosome next to the barrier (Zhang et al. 2011). Consistent with this model, deletion of genes encoding subunits of the ISW1 and CHD1 chromatin remodelers results in a major disruption of nucleosome phasing on yeast genes, which primarily reflects disruption of nucleosome spacing, because the position of the +1 nucleosome is unaffected (Gkikopoulos et al. 2011b). Thus, the location, stability, and footprint of barrier complexes are likely to be critical in phasing. It is intriguing that another ATPdependent chromatin remodeler, the essential RSC complex, plays a role in determining NDR size: The +1 and -1 nucleosomes shift into the NDR in RSC-depleted cells (Hartley and Madhani 2009). RSC depletion also results in a global loss of transcription by all three RNA polymerases (Parnell et al. 2008; Hartley and Madhani 2009). RSC is a member of the SWI/SNF family (Cairns et al. 1996,

This article is distributed exclusively by Cold Spring Harbor Laboratory Press for the first six months after the full-issue publication date (see http://genome. cshlp.org/site/misc/terms.xhtml). After six months, it is available under a Creative Commons License (Attribution-NonCommercial 4.0 International), as described at http://creativecommons.org/licenses/by-nc/4.0/. 
1999). In vitro, both RSC and SWI/SNF are capable of moving nucleosomes along DNA, ejecting them from DNA and forming remodeled structures, but they do not have nucleosome spacing activity (Lorch et al. 2001; Saha et al. 2002; Shukla et al. 2010). RSC is necessary (but not always sufficient) for positioning nucleosomes correctly in a yeast extract (Wippo et al. 2011). In vivo, both SWI/SNF and RSC bind most strongly to the $+1,+2$, and +3 nucleosomes (Yen et al. 2012).

Here we have investigated the roles of SWI/SNF and RSC in genome-wide nucleosome phasing, using paired-end sequencing to obtain high resolution nucleosome maps (Cole et al. 2012a,b). We find that SWI/SNF has no discernible influence on phasing at the global level. In contrast, RSC has a major effect on both phasing and positioning. We confirm that the flanking nucleosomes shift toward the NDR in the absence of RSC (Hartley and Madhani 2009). We show that, in RSC-depleted cells, both the upstream and downstream nucleosomal arrays shift toward the TSS with no change in spacing. Thus, RSC depletion results in a global movement of nucleosomes with respect to genes. Analysis of gene pairs in divergent, tandem, or convergent orientations reveals that nucleosome phasing patterns reflect interference between phasing signals emitted from neighboring promoters. Our observations demonstrate the critical role of RSC in organizing yeast chromatin.

\section{Results}

To deplete cells of RSC, we used a strain in which the essential RSC8 gene is under the control of the GAL1 promoter (Treich and Carlson 1997). The Rsc8 subunit is critical for the assembly and function of the RSC complex (Da et al. 2006). We confirmed that, when switched from galactose to glucose medium, the cells eventually stop growing and become larger than normal, sometimes with multiple buds (Treich and Carlson 1997). Wild-type and GAL-RSC8 cells were grown in galactose and then switched to glucose. Nuclei were isolated from both strains and digested to mono-nucleosomes with MNase. Gel-purified nucleosomal DNA was subjected to paired-end sequencing, providing 50-nt reads from both ends of each DNA molecule. Alignment of each pair of reads to the yeast genome yields the length of each nucleosomal DNA molecule; typical length distributions with the major peak at $\sim 150 \mathrm{bp}$ were observed (Cole et al. 2011a,b).

In the absence of RSC, both the upstream and downstream nucleosomal arrays invade the NDR, with no change in nucleosome spacing

Nucleosome occupancy data were analyzed both locally and globally. For local analysis, occupancy plots were constructed by counting the number of times each chromosomal coordinate base appears in a nucleosome sequence and plotting this value along the chromosome. The data for wild-type and Rsc8-depleted cells were normalized by correcting for the total number of aligned reads (Cole et al. 2011a, 2012a). A segment of chromosome II that includes the REB1, REG2, RFS1, and YBRO35C genes is illustrative (Fig. 1). In wild-type cells, there were clear NDRs at all three intergenic regions, separated by quite regular nucleosome peaks. In contrast, in Rsc8-depleted cells, the NDR between REB1 and REG2 narrowed slightly, whereas the NDRs between RFS1 and REG2 and between YBRO35C and RFS1 were filled in. An intermediate example, involving partial filling of an NDR, was observed between NHP6A and SMK1 (Fig. 1).

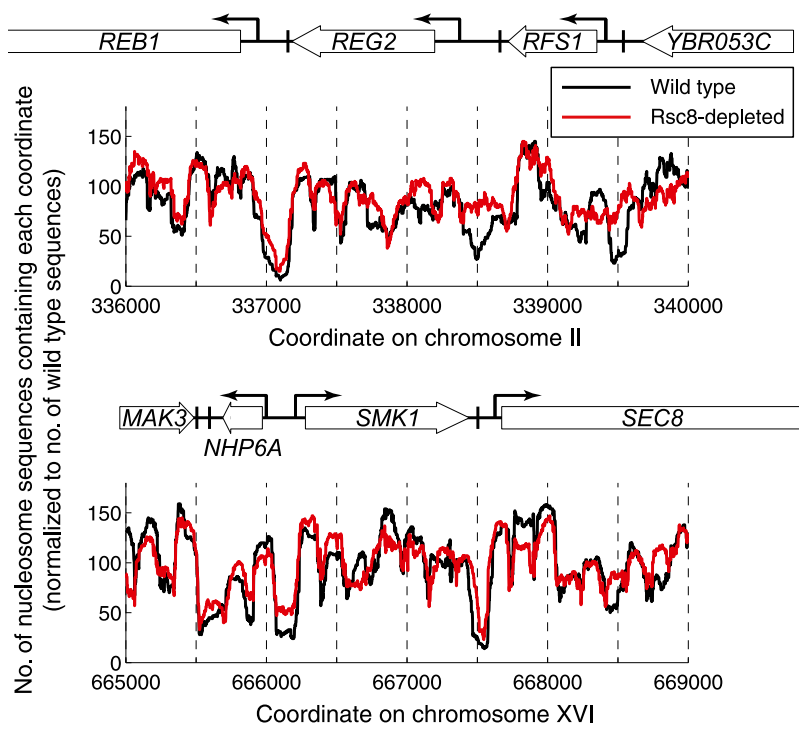

Figure 1. Nucleosome-depleted regions (NDRs) are narrower or partly filled in Rsc8-depleted cells. Nucleosome occupancy maps for two typical genomic loci illustrate narrowing and filling of NDRs. The data for Rsc8depleted cells were normalized to those for wild-type cells to adjust for the different total number of nucleosomes sequenced for the two samples.

Nucleosomes on yeast genes are phased with respect to the TSS (Yuan et al. 2005; Mavrich et al. 2008). To perform a global phasing analysis, the chromosomal coordinate of the midpoint or dyad of each nucleosome sequence in the range 140-160 bp was calculated. The number of midpoints obtained at each gene coordinate relative to the TSS was summed for all genes and plotted against the distance from the TSS. To facilitate comparison of different samples, values were normalized to the average value for each data set. Wild-type cells exhibited very clear phasing with respect to the TSS (Fig. 2A). As expected, there was a deep trough in nucleosome occupancy in the promoter region, corresponding to the NDR, with phased nucleosomes upstream and downstream. The downstream nucleosome array showed strong phasing with nucleosome peaks gradually decreasing in amplitude, beginning with the +1 nucleosome. The upstream array was less pronounced but at least four clear peaks were apparent.

Regression analysis using the peak values corresponding to the +1 to +5 nucleosomes (the downstream array) yielded a straight line with a slope of $165 \mathrm{bp}$ ( $\mathrm{SD}=1 \mathrm{bp}$; three data sets), which is equivalent to the average nucleosome spacing (Fig. 2B). This value is consistent with previously reported values of $160-165$ bp (Mavrich et al. 2008; Gkikopoulos et al. 2011b; Cui et al. 2012) and with the nucleosomal repeat length in yeast (Thomas and Furber 1976; Lohr et al. 1977). Regression analysis of the -1 to $-4 \mathrm{nu}-$ cleosomes indicated a slight increase in spacing (171 $\pm 1 \mathrm{bp} ; n=3)$. The dyad of the +1 nucleosome was located at +45 on average, indicating that its upstream boundary was at about -28 , given that the nucleosome contains $147 \mathrm{bp}$, and confirming that the TSS is located within the +1 nucleosome. The average location of the -1 nucleosome dyad was at -239 , indicating that its downstream boundary was at about -166 . Thus, the average NDR spans $\sim 138$ $\mathrm{bp}$, slightly less than the size of a nucleosome.

Nucleosome phasing in Rsc8-depleted cells was different from that in wild-type cells (Fig. 2A): All of the nucleosome peaks in the upstream and downstream arrays shifted toward the TSS. In addition, the trough representing the NDR was significantly narrower 
A
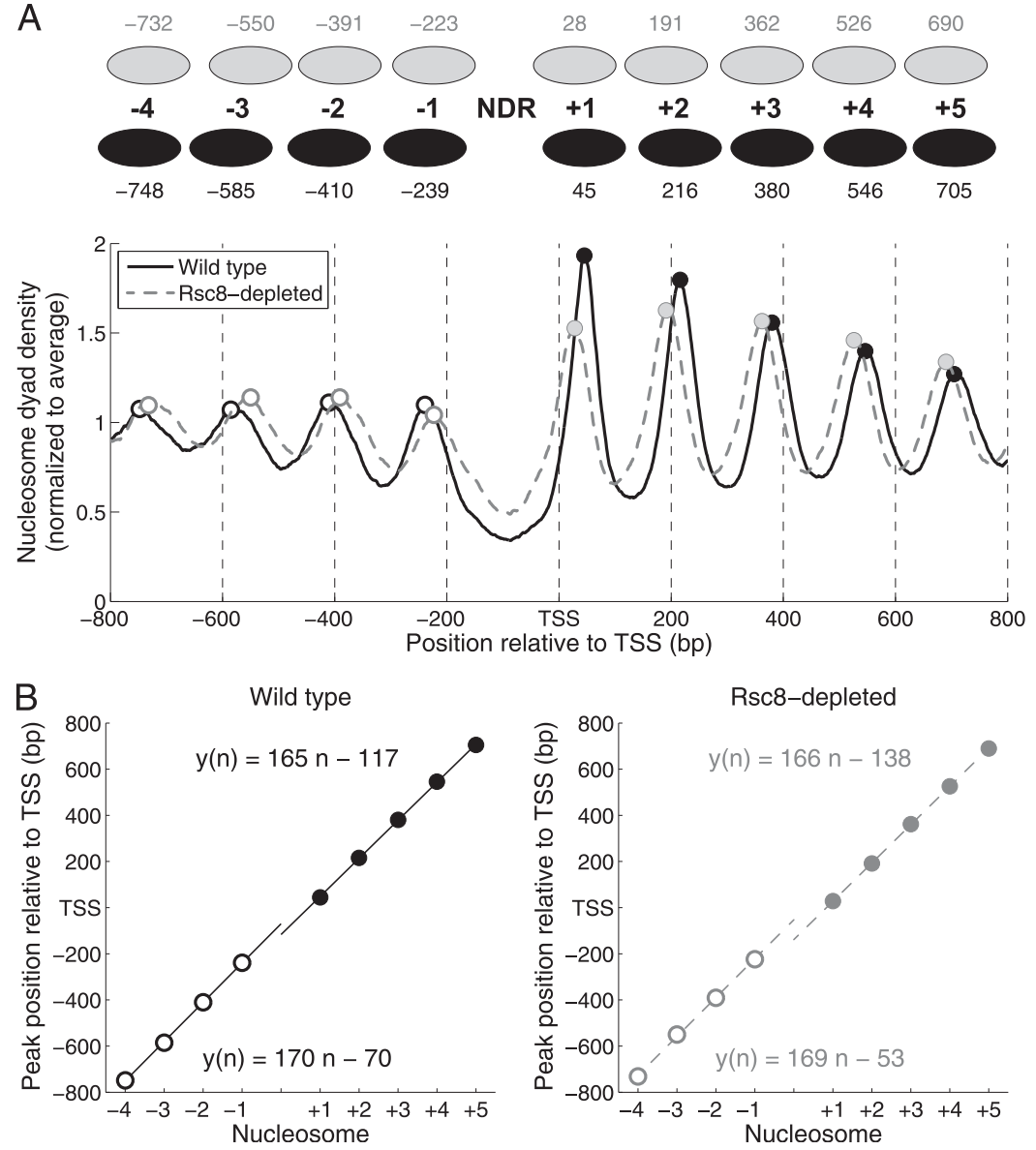

Figure 2. Nucleosome phasing relative to the transcription start site (TSS) shows global shifts of both upstream and downstream nucleosomal arrays toward the NDR in Rsc8-depleted cells, with no significant change in nucleosome spacing within each array. $(A)$ Average dyad density near the TSS in wildtype (solid line) and Rsc8-depleted cells (dashed line). The average profiles were smoothed using a moving average filter with a span of $21 \mathrm{bp}$. The first four maxima upstream of and the first five maxima downstream from the TSS are marked by open and filled circles, respectively. The midpoints (dyads) of all nucleosome sequences between 140 and $160 \mathrm{bp}$ were determined with respect to the TSS and summed for all genes. The data were normalized internally to the average value for each data set. (B) Linear functions fitted to the locations of the upstream and downstream maxima for wild-type (solid lines) and Rsc8-depleted cells (dashed lines) from A. Downstream array (filled circles); upstream array (open circles). The slope of each line gives the average distance between nucleosomes. Coefficients of determination, $\mathrm{R}^{2}$, were $>0.999$.

and shallower, consistent with the increased occupancy of some NDRs (Fig. 1), and a slight shoulder appeared on the -1 nucleosome peak, hinting at the presence of a minor peak at about -150 , within the NDR.

Relative to wild type, the +1 nucleosome midpoint shifted 17 bp (from +45 to +28 ) and the -1 nucleosome midpoint shifted 16 bp (from -239 to -223 ) on average (Fig. 2A). Thus, the average span of the NDR decreased by $\sim 33 \mathrm{bp}$, from $\sim 138$ bp in wild-type cells to $\sim 105 \mathrm{bp}$ in Rsc8-depleted cells. The regression lines for the upstream and downstream arrays in Rsc8-depleted cells had similar slopes (167 $\pm 1 \mathrm{bp}$ and $166 \pm 1 \mathrm{bp}$, respectively; $n=3$ ) to those observed for wild-type cells, indicating that the nucleosome spacing in these arrays did not change significantly (Fig. 2B). In conclusion, Rsc8 depletion results in a global shift of nucleosomal arrays into the promoter, reducing the average span of the NDR to much less than the size of a nucleosome. The upstream and downstream arrays retain the same average spacing despite the phase shift. Thus, RSC sets the phase of both the upstream and downstream nucleosomal arrays, as well as the size of the NDR.

\section{Different classes of altered chromatin structure in Rsc8-depleted cells}

The global phasing analysis (Fig. 2A) represents the average chromatin structure of all genes. To assess how typical and general the observed changes in chromatin structure in Rsc8-depleted cells are, we determined the variation in the extent to which the NDR is filled, and in the movement of the +1 and -1 nucleosomes.

In the first analysis, genes were sorted for the change in average NDR occupancy in Rsc8-depleted cells relative to wild type (Fig. 3A). The NDRs that showed the greatest increase in occupancy upon RSC depletion are at the top of the heat map; those showing the reverse trend are at the bottom. NDR occupancy was generally higher in Rsc8-depleted cells than in wild-type cells, showing that the global average is not misleading. The trends were quantified by TSS-phasing analysis of the top, central, and bottom gene tertiles (Fig. 3B). Phasing on genes in the central tertile was similar to that of the average in Rsc8-depleted cells (Fig. 2A): The nucleosome arrays shifted into the NDR, which was shallower. The upper tertile, corresponding to the genes showing the greatest increase in NDR occupancy, displayed a slightly greater shift of the downstream array into the NDR ( $\sim 10$ bp additional shift) and the upstream array shifted $\sim 7$ bp less than the other tertiles. In the upper tertile, the NDR was filled to the same depth as the troughs representing linker DNA between phased nucleosomes on the coding region, resulting in an almost continuous array, although the distance between the +1 and -1 nucleosomes was still much longer than a typical linker. The absence of a peak in the NDR indicates that the increased occupancy is not simply due to the insertion of a nucleosome over the NDR, at least at the majority of promoters, but instead reflects increased overlap of the +1 and -1 nucleosome peaks. The bottom tertile exhibited the same array shifts observed for the central tertile, combined with a shallow NDR. These genes also had a shallow NDR in wild-type cells, accounting for the small change in NDR occupancy. Importantly, there was no change in nucleosome spacing in any of the tertiles. In general, genes with deep NDRs in wild-type cells were partially but not completely filled in Rsc8-depleted cells and NDR filling reflected encroachment by flanking nucleosomes, rather than insertion of an additional nucleosome at the NDR.

In the second analysis, each gene was classified according to the change in location of its +1 and -1 nucleosomes after Rsc8 

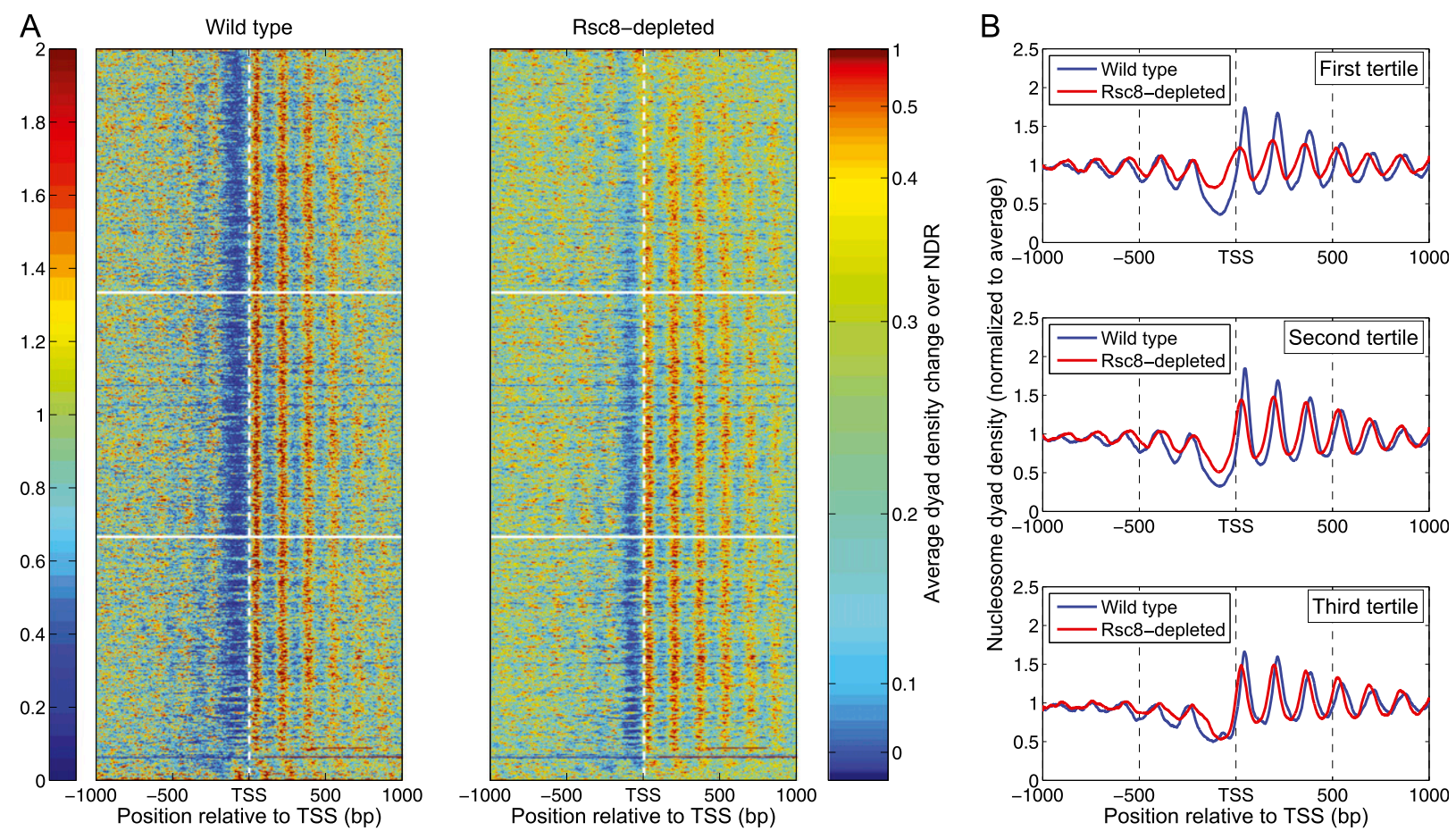

Figure 3. Rsc8 depletion results in a general increase in nucleosome density at the NDR. ( $A$ ) Heat map analysis of all genes, sorted for change in NDR occupancy. The heat maps show the nucleosome dyad distributions in a 2-kb window centered on the TSS. Each row represents a gene. The genes were sorted according to the average change in dyad density in the NDR, which was defined as the region from -150 to -50 relative to the TSS (right color bar). Genes displaying the largest increase in nucleosome dyad density in the NDRs of Rsc8-depleted cells relative to wild type are at the top. The heat maps were smoothed with a 2D symmetric Gaussian filter with standard deviation $\sigma=3$, the color plot representing the average dyad density change was smoothed using a moving average filter with a span of 21 genes. (B) Average nucleosome dyad density profiles for the top, central, and bottom gene tertiles, delimited by the white horizontal lines in $A$. The plots were smoothed using a moving average filter with a span of $21 \mathrm{bp}$.

depletion. There are nine possible classes, because each of the two nucleosomes can either shift upstream or downstream, or stay in place. For all genes, the shift of the +1 nucleosome was plotted against the shift in the -1 nucleosome (Fig. 4A). The largest fraction of genes (33\%) showed the expected shift of the +1 and -1 nucleosomes into the NDR (gene cluster 1). The second largest set of genes (cluster 4 ) showed a shift of the +1 nucleosome into the NDR with no change in the -1 nucleosome (21\%) and, on an additional $7 \%$ of genes, the +1 nucleosome shifted into the NDR while the -1 nucleosome shifted away from the NDR (cluster 7 ). In the case of the -1 nucleosome, $12 \%$ of genes showed a shift into the NDR with no change in the +1 nucleosome (cluster 2), and 2\% showed a shift into the NDR combined with a shift of the +1 nucleosome away from the NDR (cluster 3 ). Another $13 \%$ of genes showed little or no change in the positions of the +1 and -1 nucleosomes (cluster 5 ). Thus, the average change in the locations of the +1 and -1 nucleosomes masks a variety of effects, although $75 \%$ of genes showed at least one of the nucleosomes shifting into the NDR, accounting for the global picture.

Each cluster of genes was subjected to TSS-phasing analysis (Fig. 4B). The most populous cluster (cluster 1), with both +1 and -1 nucleosomes shifting into the NDR, exhibited the same phasing pattern as the average, with nucleosomal array shifts, a narrower NDR, and no change in spacing. Cluster 4 showed a strong shift in the +1 nucleosome with no change in the -1 nucleosome. Cluster 7 , in which the +1 nucleosome shifted into the NDR and the -1 nucleosome shifted away from it, was more ambiguous, because the -1 nucleosome appeared to be missing, with the -2 and -3 nucleosomes shifting toward the NDR. Cluster 2 genes displayed a weak peak within the NDR in wildtype cells; in Rsc8-depleted cells, the NDR was partially filled, the weak peak was absent, and there was poor phasing upstream. Genes in cluster 8 did not have a clear NDR and exhibited poor phasing in wild-type and Rsc8-depleted cells. Cluster 3 showed complete filling of the NDR in Rsc8-depleted cells and a complete loss of phasing, but it comprised only $2 \%$ of genes. Clusters 6 and 9 showed striking phase shifts in Rsc8-depleted cells, such that the NDR was partly or completely occupied by a nucleosome, although together they account for only $5 \%$ of genes. In summary, the large majority of genes showed a shift of the +1 or the -1 nucleosome into the NDR; a minority of genes have NDRs that are filled by nucleosomes.

\section{RSC binds preferentially to heavily transcribed coding regions}

To address the question of how RSC-mediated changes in chromatin structure relate to transcription, we sorted the genes according to their relative expression, as measured by the amount of Pol II on the coding region in wild-type cells (Fig. 5A; data of Elfving et al. 2014). These Pol II ChIP data correlated well with mRNA measurements reported by Holstege et al. (1998), as expected. In wild-type cells, the most highly transcribed genes (at the top of the heat map) showed wider NDRs and poor phasing on their coding regions, as observed previously (Ioshikhes et al. 2006; Zawadzki et al. 2009; Weiner et al. 2010). This was also true in Rsc8depleted cells, although the poor phasing over the coding region was still accompanied by a general nucleosome shift toward the 
A

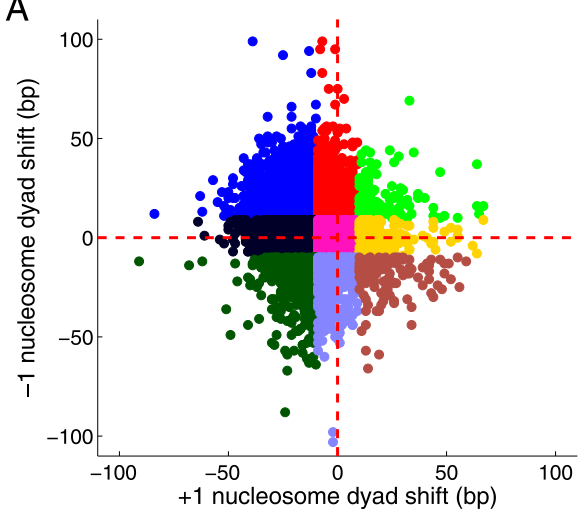

Number of genes

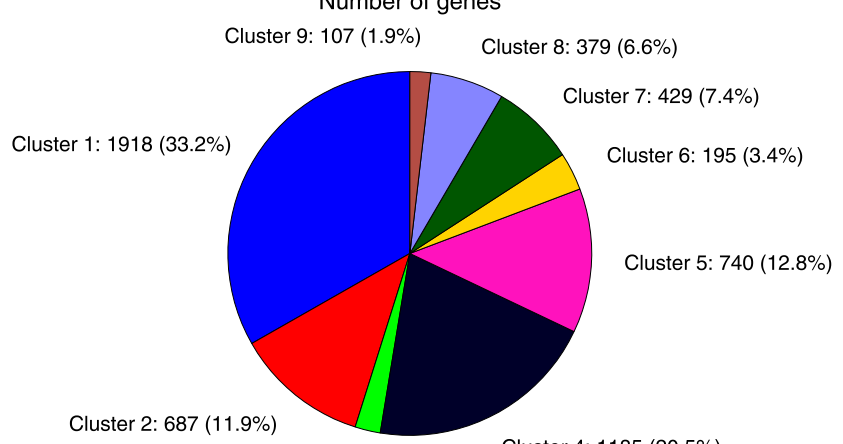

Cluster 3: $130(2.3 \%)$
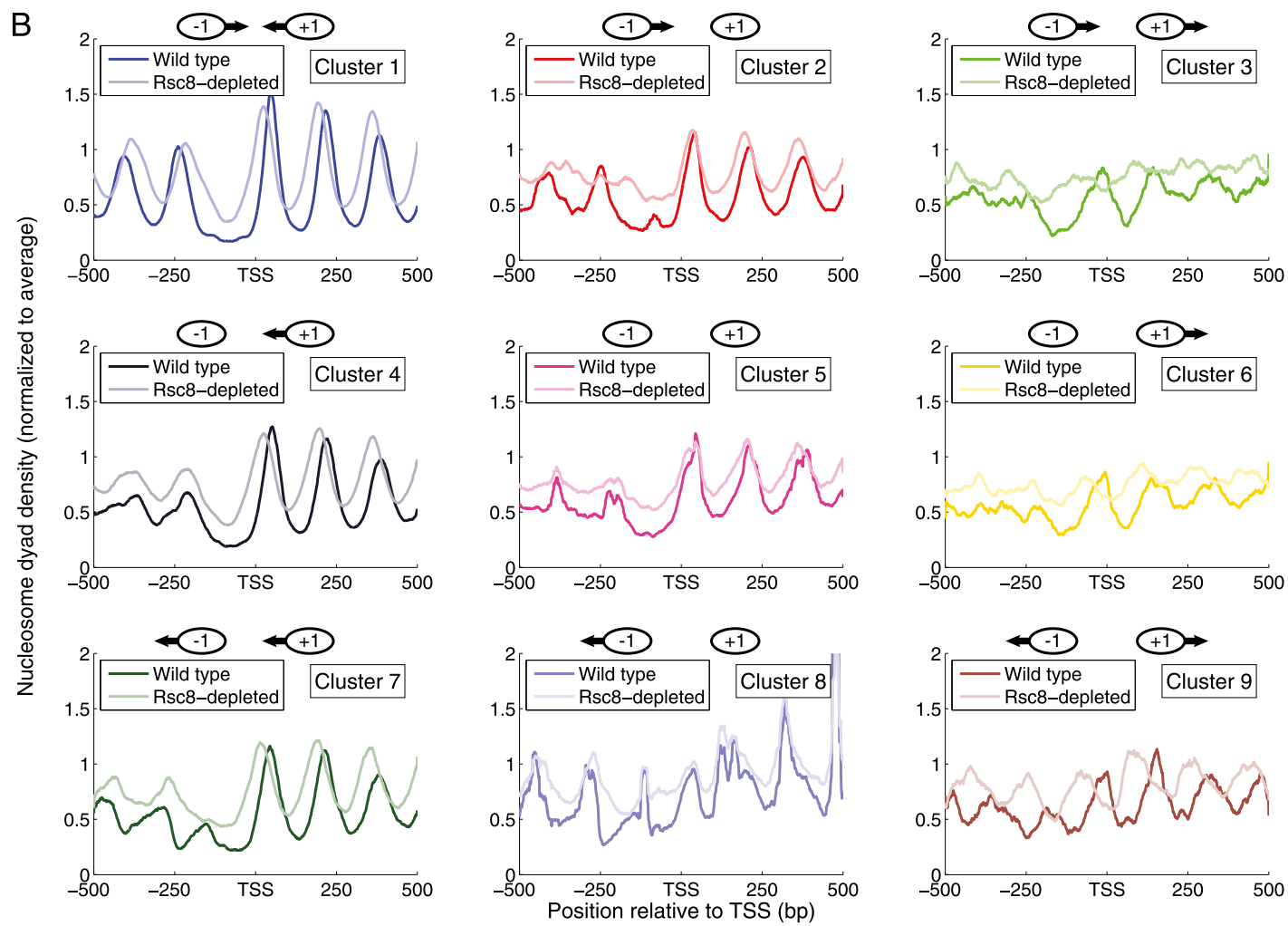

Figure 4. Different classes of altered chromatin structure in Rsc8-depleted cells. $(A)$ Scatter plot representing the shift in the +1 nucleosome dyad versus the shift in the -1 nucleosome dyad for each gene in Rsc8-depleted cells relative to wild type. A negative shift indicates an upstream movement of the nucleosome in Rsc8-depleted cells. The genes are color-coded to define nine clusters of genes, according to whether the +1 and -1 nucleosomes shift upstream or downstream, or remain in place (shift $<10 \mathrm{bp}$ ). The pie chart indicates the fraction and number of genes in each cluster. (B) Average nucleosome dyad density profiles in a 1-kb window centered on the TSS for each of the nine gene clusters defined in $A$. The profiles were smoothed using a moving average filter with a span of $21 \mathrm{bp}$. The cartoon at the top of each panel indicates the direction of displacement of the +1 and -1 nucleosomes.

NDR. Thus, the wide NDRs associated with highly active genes are preserved in Rsc8-depleted cells.

To determine whether there is any correlation between RSC binding (data of Floer et al. 2010) and transcription, the relative amounts of RSC on the NDRs and coding regions of these genes were plotted in the same order (Fig. 5A). Although there was only a weak correlation between transcription and RSC binding at NDRs, there was a good positive correlation between the amount of RSC on the coding region and transcription. Thus, RSC binds preferentially to the coding regions of highly transcribed genes. The chromatin structures of these genes were similar in wild-type and Rsc8-depleted cells (both showed wide NDRs and poor nucleosome phasing), except that the characteristic shift in the nucleosomal array toward the promoter was still observed in Rsc8-depleted cells.

Increased nucleosome occupancy of NDRs in Rsc8-depleted cells does not correlate with RSC binding in wild-type cells

It might be expected that those NDRs most affected by RSC would be associated with high levels of RSC. Accordingly, we addressed the relationship between increased nucleosome occupancy of the NDR in Rsc8-depleted cells and RSC binding in wild-type cells (data of Floer et al. 2010). The genes were sorted according to change in NDR occupancy (Fig. 5B). However, there was no correlation between 
A

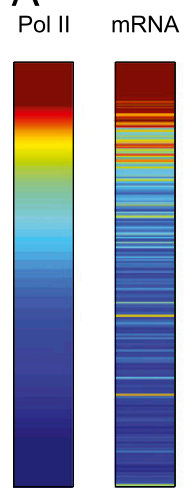

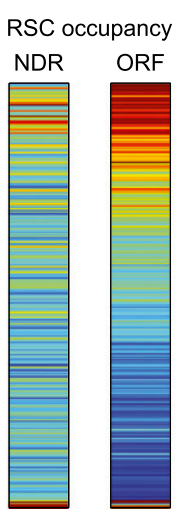

Nucleosome dyad density

Rsc8-depleted

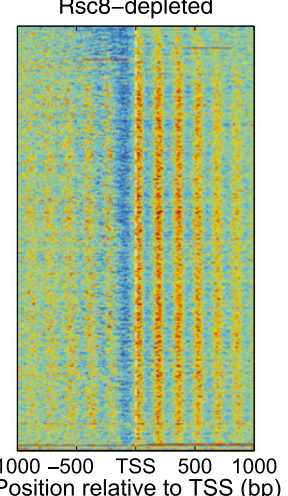

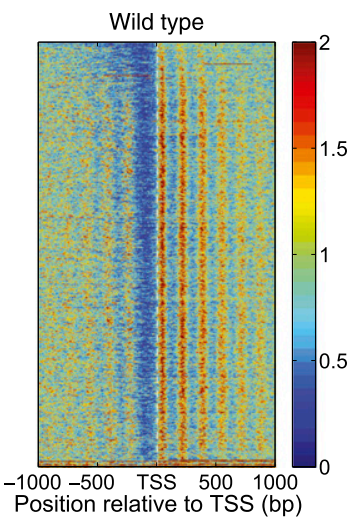

B

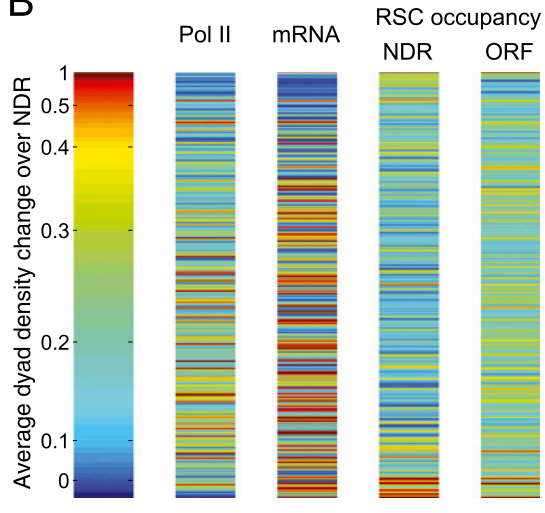

Figure 5. Preferential binding of RSC to heavily transcribed coding regions. ( $A$ ) Correlation between Pol II density and RSC binding to coding regions. Genes were sorted according to their average ChIP signal for the CTD of Pol II over the transcription unit (left panel) (data from Elfving et al. 2014), such that the genes with the highest Pol II density are at the top. Expression data (Holstege et al. 1998), RSC occupancy data (ChIP for TAP-tagged Rsc8) at NDRs or coding regions (Floer et al. 2010), and our nucleosome dyad phasing data for wild-type and Rsc8-depleted cells are plotted in the same gene order. (B) Rsc8 depletion has the strongest effect on the NDRs of weakly transcribed genes. Genes were sorted according to increase in average NDR dyad density in Rsc8-depleted cells relative to wild type, such that the most affected genes are at the top (sorted as in Fig. 3A). Pol II density, expression data, and RSC occupancy data for the NDR and coding regions are plotted in the same gene order. Heat maps and plots were smoothed as described in the legend to Figure 3.

increased NDR occupancy and RSC binding at the NDR or on the coding region. There was also no clear correlation between the gene clusters defined by the directional shifts of the +1 and -1 nucleosomes (see Fig. 4) and RSC binding (Supplemental Fig. S1).

We also asked whether those genes with NDRs showing the largest increase in nucleosome occupancy in Rsc8-depleted cells correlate with transcriptional level (data of Holstege et al. 1998; Elfving et al. 2014). Generally, there was little correlation between transcription and the extent to which the NDR was filled, except that the $\sim 350$ most affected genes (at the top of the heat map) tended to be weakly expressed in wild-type cells (Fig. 5B). There was also no clear correlation between the gene clusters defined by the directional shifts of the +1 and -1 nucleosomes (see Fig. 4 ) and transcriptional activity (Supplemental Fig. S1; Holstege et al. 1998; Elfving et al. 2014).

\section{Unlike RSC, SWI/SNF does not have global effects on promoter occupancy or nucleosome phasing}

In yeast, the ATP-dependent chromatin remodeling complex most similar to RSC is SWI/SNF. To assess whether our observations are specific to RSC or more generally true of this type of complex, we analyzed the chromatin structure of snf $2 \Delta$ cells using the same approach (SNF2 encodes the ATPase subunit of SWI/SNF). In contrast to our observations for Rsc8-depleted cells, snf2A cells did not differ significantly from wild-type cells in nucleosome phasing or spacing with respect to the TSS (Supplemental Fig. S2A).

To determine whether there are classes of genes with altered chromatin structure that are masked by the global average, we subjected the snf $2 \Delta$ data to NDR occupancy analysis. As described above for Rsc8-depleted cells, genes were sorted for the change in average NDR occupancy in $s n f 2 \Delta$ cells relative to wild type; the resulting heat maps are similar to one another, indicating that $s n f 2 \Delta$ did not have a major effect (Supplemental Fig. S2B). The wild-type heat maps obtained after sorting for change in NDR occupancy relative to snf2s cells (Supplemental Fig. S2B) and Rsc8-depleted cells (Fig. 3A) differ in appearance: Genes with wide NDRs and poor phasing were the most affected in snf $2 \Delta$ cells; these are attributes of highly transcribed genes. However, TSS-phasing analysis of the gene tertiles indicated that the differences between wild-type and $s n f 2 \Delta$ cells are minor (Supplemental Fig. S2C). Strong phasing was observed in all cases, with no change in nucleosome spacing. We conclude that SWI/SNF is not a major player in chromatin organization at the global level in yeast.

\section{Nucleosome phasing is bidirectional}

The shift of the upstream nucleosomal array toward the NDR in Rsc8-depleted cells poses a conundrum concerning the orientation of the gene upstream: If the upstream gene is divergent with respect to the downstream reference gene (i.e., the gene whose TSS is under consideration), then its nucleosomal array is also expected to shift toward the promoter, as observed. However, if the upstream gene is transcribed in the same direction as the reference gene (tandem orientation), then the prediction is ambiguous because its nucleosomal array might shift upstream toward its own promoter or downstream toward the reference TSS.

To address this issue, nucleosome phasing analysis was performed on genes sorted according to whether the upstream gene is divergent or tandem with respect to the downstream reference gene (Fig. 6). Because wider NDRs will reduce the amplitudes of the upstream nucleosome peaks, particularly the -1 and -2 nucleosomes, divergent and tandem genes were first sorted according to the size of their intergenic regions. For divergent genes, inter-TSS distances were calculated and plotted as a histogram (Fig. 6A). Remarkably, the distribution was not random, but instead composed of a major, fairly narrow, peak at $\sim 190 \mathrm{bp}$, ranging from $\sim 70$ to $\sim 270 \mathrm{bp}$, with a tail to much higher values.

Upstream nucleosome phasing on the subset of divergent genes with inter-TSS distances of $<270$ bp was much improved in both wildtype and Rsc8-depleted cells (Fig. 6B), relative to upstream phasing on all genes (Fig. 2A). In fact, upstream phasing for short divergent promoters was nearly as good as downstream phasing, as judged by the relative amplitudes of the dyad peaks and the number of clear nucleosome peaks observed (Fig. 6B). As expected, this improvement in phasing is partly due to the removal of divergent genes with intergenic regions $>270 \mathrm{bp}$, as shown by the much weaker upstream phasing detected on the genes in this category (Supplemental Fig. S3).

\section{Genome Research} www.genome.org 

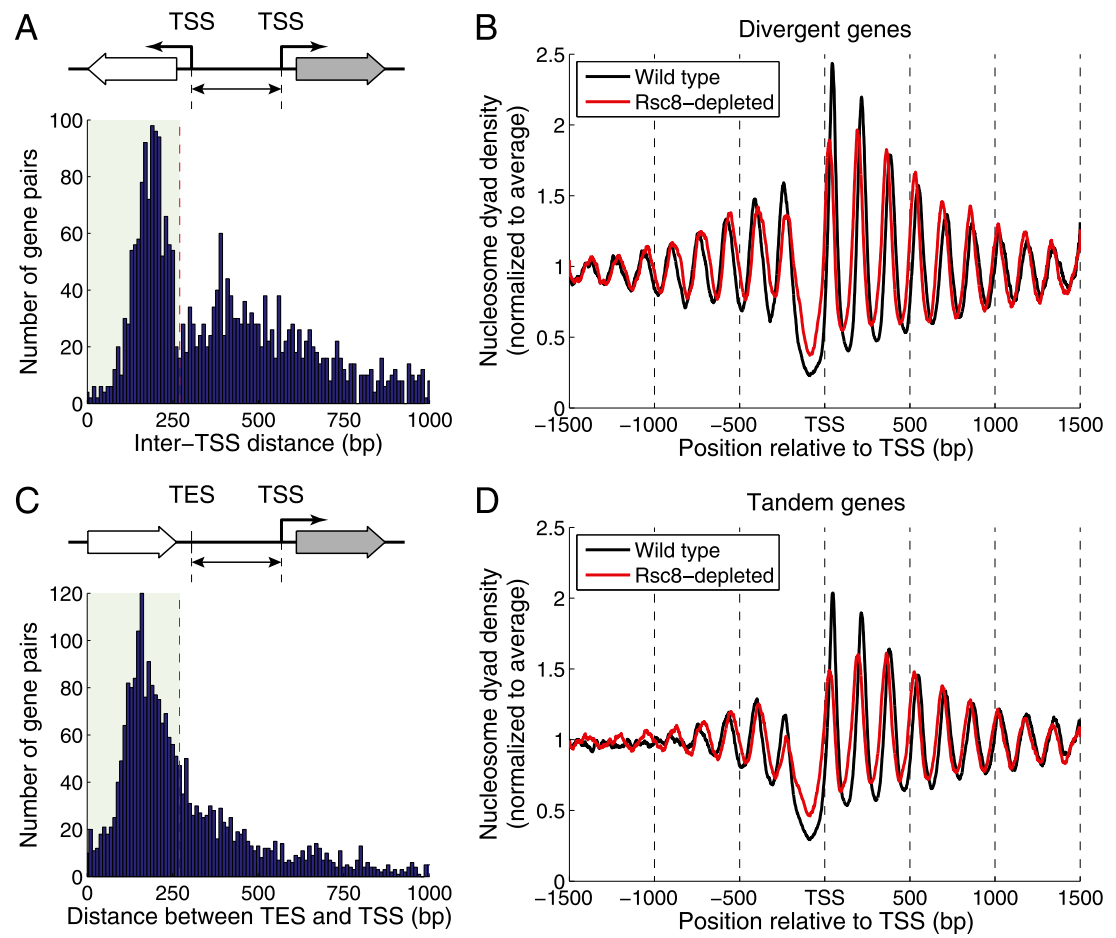

Figure 6. Upstream nucleosomes in Rsc8-depleted cells shift toward the NDR regardless of the orientation of the upstream gene. ( $A$ ) Histogram of inter-TSS distances for divergent genes. (B) Average nucleosome dyad density profiles for divergent genes with inter-TSS distances $<270 \mathrm{bp}$ (shaded box in $A$ ). (C) Histogram of distances between TES and TSS for tandem genes. (D) Average nucleosome dyad density profiles for tandem genes with TES-TSS distances $<270$ bp (shaded box in C). In $B$ and $D$, gene pairs are aligned to the TSS of the downstream gene.

Tandem genes have a reference TSS with a transcript end (termination) site (TES) upstream (Fig. 6C). First, we examined the possibility that the TES located between tandem genes acts as a phasing barrier between the two genes, such that nucleosomes upstream of the TES are shifted toward the upstream TSS and nucleosomes downstream from the TES shift toward the downstream reference TSS. If so, the TES would be expected to have some phasing properties. However, although the NDR associated with the TES was detected, the TES had only very weak phasing properties in wild-type or Rsc8-depleted cells (Supplemental Fig. S4), consistent with a previous report (Mavrich et al. 2008).

To estimate the size of the intergenic region between tandem genes, we calculated the distance between the TES and the TSS (Fig. 6C). Again the distribution was not random, displaying a peak at $\sim 120 \mathrm{bp}$ and tailing off to high values. Phasing analysis for tandem genes with TES-TSS distances of $<270 \mathrm{bp}$ showed clear but weaker upstream phasing relative to divergent genes (Fig. 6B,D). Nucleosome spacing and locations on divergent and tandem genes were very similar to those observed for all genes in both wild-type and Rsc8-depleted cells. Although phasing on tandem genes was weaker than on divergent genes, the important observation is that the $-1,-2$, and -3 nucleosomes (defined by the downstream TSS) of a tandem gene pair still shifted toward the downstream promoter in Rsc8-depleted cells (Fig. 6D). Phasing analysis of the upstream genes in tandem gene pairs (i.e., using the upstream TSS as the reference gene) indicated that the nucleosomes shift toward its TSS (Supplemental Fig. S3). Therefore, nucleosomes on the upstream gene of a tandem gene pair appear to shift in both directions-toward its TSS and away from it, toward the downstream
TSS. Thus, nucleosome phasing relative to the TSS is bidirectional, acting both upstream and downstream.

\section{Convergent gene pairs exhibit constructive and destructive interference between opposing nucleosomal arrays}

If nucleosomes are indeed phased both upstream and downstream with respect to the reference TSS (i.e., bidirectionally), then the DNA between neighboring promoters will be subject to nucleosome phasing signals from both promoters, independently of gene orientation. If the length of the intervening DNA is not equal to an integral number of nucleosomal repeats $(\sim 165 n$, where $n$ is the number of nucleosomal repeats), then the two phasing signals will interfere with one another, disrupting the phasing pattern. If, on the other hand, the distance between the promoters is approximately equal to $165 n$, then constructive interference will occur and a very regular nucleosomal array should be apparent. More broadly speaking, we propose that the typical phasing pattern is the product of constructive or destructive interference between overlapping arrays emanating from neighboring phasing points. Interference would tend to broaden the peaks and reduce their amplitude and new sub-peaks might appear if the phase difference is sufficiently large.

The TSS is a phasing reference point but it is unlikely to be directly responsible for phasing because it is located within the +1 nucleosome, which is shifted in RSC-depleted cells. Instead, the actual phasing point is likely to be the downstream border of a barrier located at the NDR that is a fixed distance from the TSS. Since we have no reference point for the upstream boundary of the barrier, we cannot analyze phasing interference on tandem or divergent gene pairs. Therefore, to test the concept of interference between phased arrays, we analyzed convergent gene pairs, which have two TSS phasing points directed toward one another (Fig. 7). Convergent gene pairs separated by possible phasing elements such as tRNA genes (Mavrich et al. 2008; Nagarajavel et al. 2013), centromeres, or replication origins were excluded.

Initially we examined the TSS-phasing patterns of nucleosomes on convergent gene pairs sorted by inter-TSS distance (Fig. 8A). In both wild-type and Rsc8-depleted cells, phasing was strong near both TSSs but weaker in between, suggesting that phasing power decreases with distance from the TSS (the likely reason for this is discussed below). The predicted interference patterns between the TSSs would be difficult to discern in the heat maps because nucleosomes should cycle in and out of phase through every 165-bp increment in inter-TSS distance; however, there are not enough gene pairs of similar lengths in most regions to test this systematically.

To circumvent this problem, we divided the genes into four sub-groups according to their predicted phase difference relative to the nucleosomal repeat of $165 \mathrm{bp}$ in wild-type cells and $166 \mathrm{bp}$ in 
A

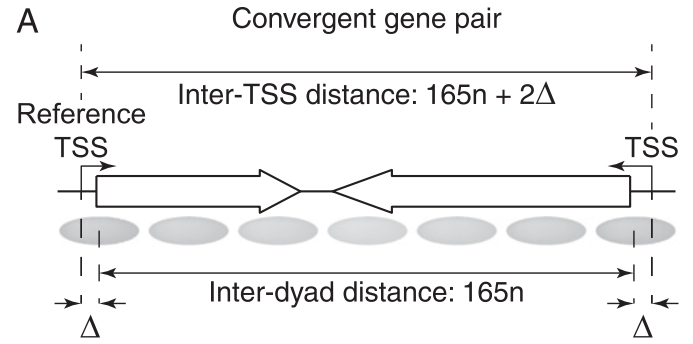

B

In phase arrays of nucleosomes Constructive interference

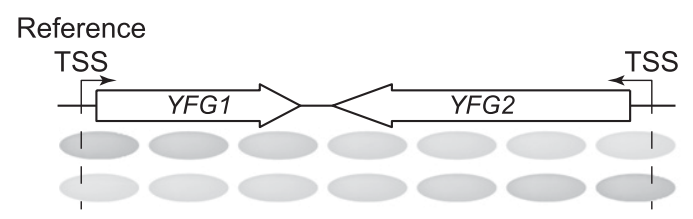

Out of phase arrays of nucleosomes Destructive interference

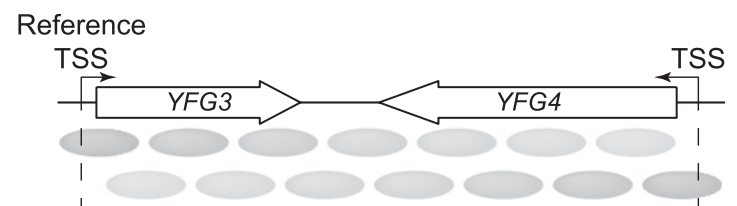

Figure 7. Phase interference model applied to convergent gene pairs. $(A)$ A nucleosomal array formed on the DNA between convergent TSSs. If a regular array of nucleosomes has a repeat length of $165 \mathrm{bp}$ and the dyads of the +1 nucleosomes at both ends of the array are located at a distance $\Delta$ downstream from the TSS, then the inter-TSS distance is $165 n+2 \Delta$, where $n$ is an integer equal to the number of nucleosomal repeats $(\Delta=45$ or $28 \mathrm{bp}$ for wild-type and Rsc8-depleted cells, respectively; see Fig. 2B). (B) Constructive interference: nucleosomal arrays phased relative to both TSSs are in phase. Destructive interference: the nucleosomal array phased relative to the reference TSS is out of phase with the array phased relative to the other TSS. The relative phase of the two arrays depends on the inter-TSS distance.

Rsc8-depleted cells (Fig. 2B), as follows: the number of nucleosomal repeats that fit between two TSSs, $n=(d-2 \Delta) / 165$, where $d$ is the inter-TSS distance and $\Delta$ is the distance between the TSS and the dyad of the +1 nucleosome ( $\Delta$ takes into account the fact that the TSS is inside the +1 nucleosome) (Fig. 7A). Thus, $\Delta$ is $45 \mathrm{bp}$ for wild-type cells and $28 \mathrm{bp}$ for Rsc8-depleted cells; they differ because of the increased nucleosome shift into the NDR observed in Rsc8-depleted cells (Figs. 2A, 7A). If $n$ is an integral number, nucleosomal arrays beginning at both TSSs should be in phase and constructive interference is predicted. If $n$ is nonintegral, the arrays will be out of phase, resulting in destructive interference. The phase difference is given by the decimal fraction of $n$, which translates into a fraction of a 165-bp repeat. The four groups of convergent gene pairs were defined by their phase difference $( \pm 20$ bp): $0 \mathrm{bp}$ (in phase), $40 \mathrm{bp}, 80 \mathrm{bp}$ (almost exactly out of phase), and $120 \mathrm{bp}$. Each convergent gene pair was assigned to its nearest phase group by calculating $n$ using the known value of $d$ and the appropriate value of $\Delta$ for wild-type and Rsc8-depleted cells.

Initially, the analysis was limited to all convergent gene pairs with inter-TSS distances $<3 \mathrm{~kb}$ (see blue boxes in Fig. 8A). As expected, the phasing profiles for these genes were very similar to those observed for all genes in wild-type cells, showing the same spacing of $\sim 165 \mathrm{bp}$ for the downstream array in both cases. Phasing weakened with distance from the reference TSS, decreasing to almost background after approximately seven nucleosome peaks (Fig. 8B). Critically, the phase groups showed quite different behavior, even though they contained many fewer genes, which would be expected to decrease the signal-to-noise ratio. In all phase groups, peaks that were absent in the analysis of the complete set of genes appeared near the downstream TSS. The explanation is that the new downstream peaks are at different locations in the different phase groups and so they cancel out due to interference when the complete set of genes is considered. As predicted, the nucleosomes were only in phase with the 165 -bp repeat over the entire distance between the TSSs in the group with a phase difference of $0 \mathrm{bp}$ (Fig. 8B; the dashed lines indicate 165-bp increments). Although the genes with a phase difference of $40 \mathrm{bp}$ also gave a regular array, there was a hint of peak splitting in the middle and the downstream nucleosomes were clearly out of phase with the upstream nucleosomes. Destructive interference between the two arrays was most obvious in the 80-bp phase group, where the central peaks were almost completely flattened. Essentially the same result was obtained for Rsc8-depleted cells (Fig. 8C). This represents an important test of the interference model because the genes in each phase group are different in Rsc8-depleted cells, due to the array shifts into the NDR (taken into account by the value of $\Delta$ ).

We repeated the phase difference analysis on convergent gene pairs with larger inter-TSS distances (pink boxes in Fig. 8A). The gene pairs with a phase difference of $0 \mathrm{bp}$ showed poor phasing in the central region. We attribute this effect to loss of phasing power with distance from the TSS (Supplemental Fig. S5). Thus, interference between the two arrays is minimal if the TSSs are sufficiently far apart.

\section{Modified barrier model for nucleosome phasing}

We propose an active mechanism of nucleosome positioning to explain loss of phasing with distance from the NDR: A protein complex located at the NDR directs remodeling enzymes to position the +1 nucleosome on the DNA immediately downstream from the boundary of the complex, which defines the barrier. The remodelers then act recursively to position the +2 nucleosome at a defined distance from the +1 nucleosome and then to position the +3 nucleosome relative to the +2 nucleosome etc., resulting in regular spacing. The heterogeneity in the position of the +1 nucleosome will have a "knock-on" effect on the positioning of subsequent nucleosomes, resulting in a gradual decay in phasing power. That is, the variance of the position of the $+N$ nucleosome is $N$ times the variance of the +1 nucleosome (Supplemental Material).

Specifically, we assumed that the remodelers position nucleosomes at a distance that is normally distributed around an average nucleosome spacing. We computed the distribution of dyads for all nucleosomes that are positioned downstream from the TSS, assuming that remodelers act either unidirectionally from the barrier or bidirectionally, between neighboring barriers. These distributions depend on three parameters: the position of the phasing barrier, $B$, and the two parameters of the Gaussian distribution; the average nucleosome spacing, $D$; and the standard deviation, $\sigma$. We optimized the three parameters for wild-type and Rsc8-depleted cells, such that the predicted dyad distributions fit the observed dyad distributions downstream from the TSS for long ( $>3 \mathrm{~kb}$ ) genes (Supplemental Fig. S5).

The effect of interference due to an array phased relative to a downstream barrier can be modeled, but the result depends on the phase difference between the two barriers, as discussed above. However, in the special case of long inter-TSS distances, where phasing dissipates at distances far from the TSS, the nucleosomes 
A

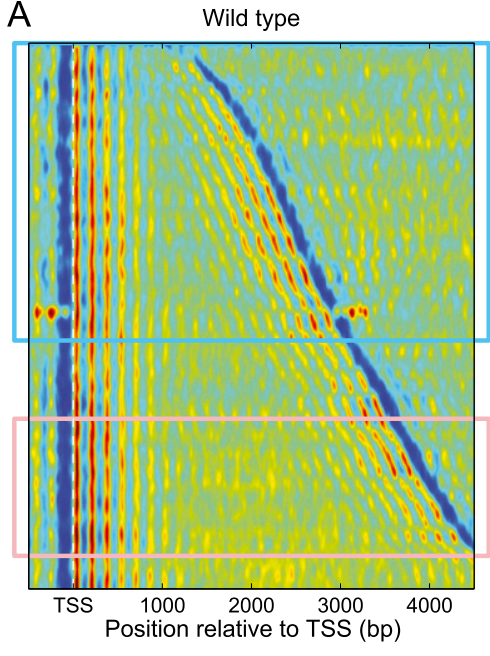

B
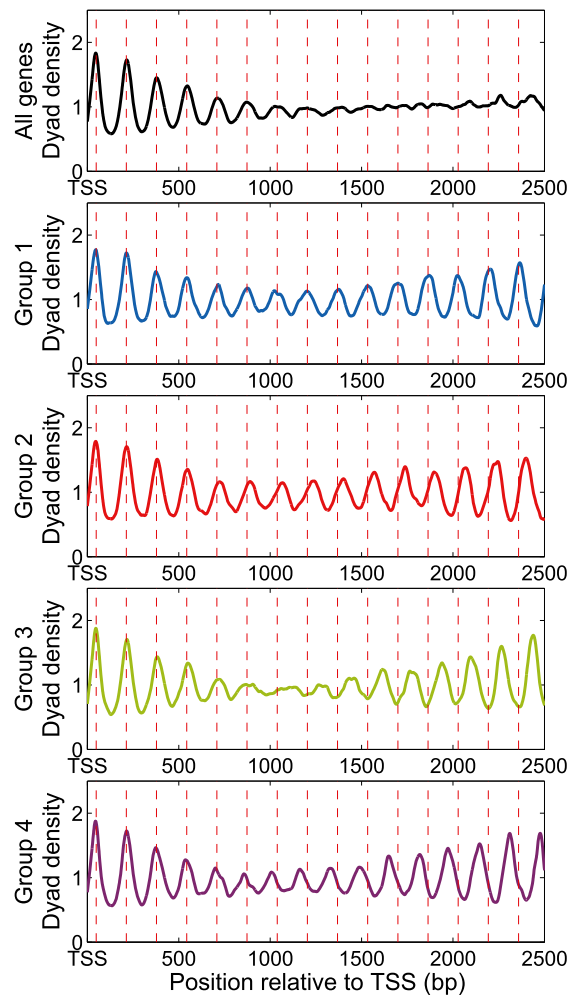

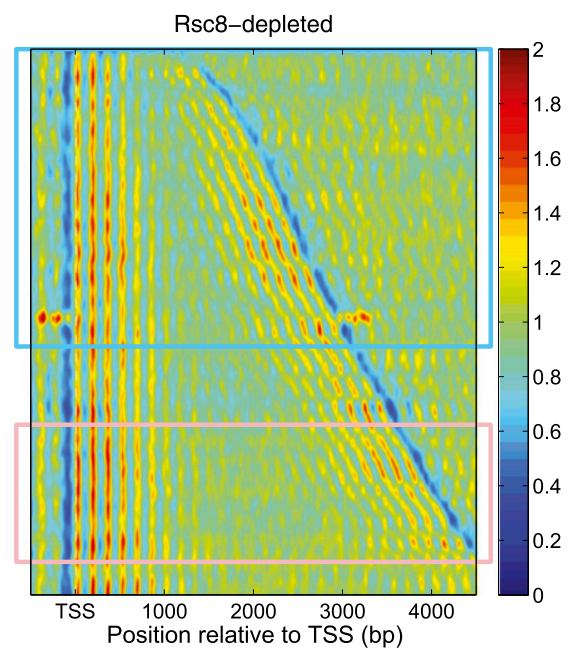

C
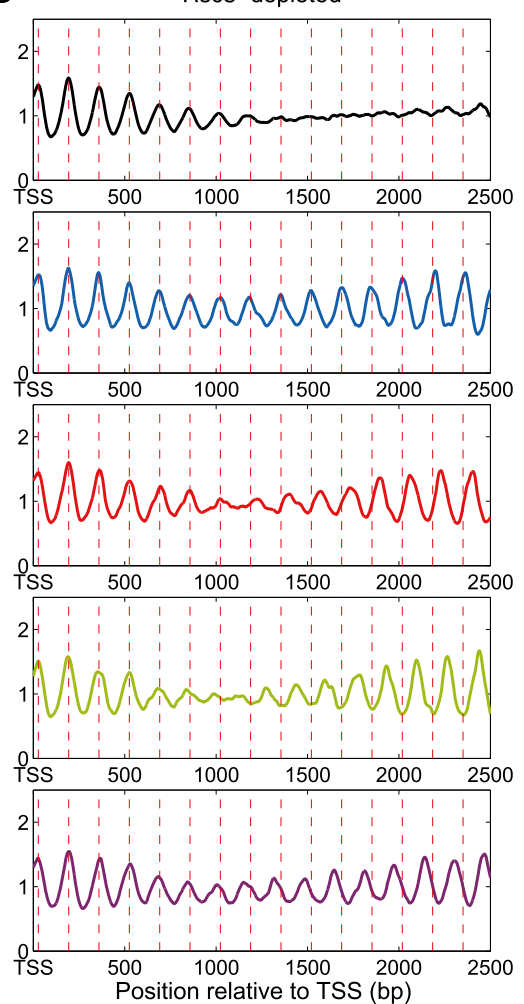

Figure 8. Phase interference analysis of convergent gene pairs. $(A)$ Heat maps representing pairs of convergent genes with inter-TSS distances $<5 \mathrm{~kb}$, in wild-type and Rsc8-depleted cells. Gene pairs containing potential phasing elements (replication origins [ARSs], centromeres, LTRs, snRNA genes, snoRNA genes, and tRNA genes) were removed from the list. Gene pairs were sorted by inter-TSS distance. Heat maps were smoothed with a 2D symmetric Gaussian filter with standard deviation $\sigma=15$. Rectangles denote convergent gene pairs with inter-TSS distance $<3 \mathrm{~kb}$ (blue rectangle) and between 3.5 and $4.5 \mathrm{~kb}$ (pink rectangle). ( $B, C$ ) Interference patterns for convergent gene pairs with inter-TSS distances $<3 \mathrm{~kb}$ (blue rectangle in $A$ ). Top panels show the average for all of these convergent gene pairs. The lower four panels correspond to four phase groups 1-4, representing gene pairs with phase differences of 0 (in phase), 40,80 (almost exactly out of phase), and $120 \mathrm{bp}$, respectively (all $\pm 20 \mathrm{bp}$ ). (B) Wild type. (C) Rsc8-depleted cells. Note that the four groups contain different genes because of the nucleosome shifts into the NDR represented by the value of $\Delta$ (Fig. 7A). The average dyad plots were smoothed using a moving average filter with a span of $51 \mathrm{bp}$.

belonging to the array originating from the downstream barrier become unphased, resulting in a uniform dyad density, 1/D (see Supplemental Methods). The fit to our data improved significantly after incorporating bidirectional phasing into the model (Supplemental Fig. S5). The nucleosome spacing parameters for wildtype and Rsc8-depleted cells were very similar ( $D \sim 165 \mathrm{bp} ; \sigma \sim 18 \mathrm{bp}$ ), whereas the predicted location of the NDR barrier boundary was shifted $\sim 20$ bp upstream in Rsc8-depleted cells. Thus, the overall fit of the model to the data was excellent.

In conclusion, analysis of convergent gene pairs supports the hypothesis that nucleosome phasing emanating from an NDR is bidirectional and subject to interference from the phasing signal arising from a neighboring NDR. The degree of interference depends on the distance between the NDRs.

\section{Discussion}

In the absence of the RSC-remodeling complex, the NDR shrinks by $\sim 33 \mathrm{bp}$. The narrowing of the NDR is a consequence of the movement of the entire upstream and downstream nucleosomal arrays toward the promoter, with no change in nucleosome spacing. It follows that a global change in nucleosome positioning occurs in RSC-depleted cells. It may be concluded that RSC is required to maintain the correct positioning of the upstream and downstream nucleosomal arrays with respect to the promoter.

Although the RSC complex catalyzes nucleosome mobilization and octamer transfer in vitro (Cairns et al. 1996; Lorch et al. 2001), its effects in vivo might not all be direct, because RSC is targeted to a large subset of genes (Damelin et al. 2002; Ng et al. 2002; Badis et al. 2008). We analyzed phasing on the RSC-target genes reported by $\mathrm{Ng}$ et al. (2002) but they did not differ significantly from nontarget genes. However, using the RSC occupancy data of Floer et al. (2010), we show here that RSC is preferentially located on the coding regions of heavily transcribed genes, rather than at their NDRs. This observation is consistent with preferential binding of RSC to the $+1,+2$, and +3 nucleosomes (Yen et al. 2012). RSC might facilitate transcription by moving or transferring nucleosomes, resulting in a general disruption in the chromatin of heavily transcribed genes (wider NDRs and relatively poor phasing). Such genes still have wider NDRs and poor phasing in Rsc8-depleted cells, indicating that RSC is not required for chromatin disruption. Transcription is not likely to be the direct cause of chromatin disruption given the low levels of transcription in RSC-depleted cells (Parnell et al. 2008; Hartley and Madhani 2009). Instead, RSC 
might restore the chromatin structures of heavily transcribed genes by reversing disruption due to transcription. RSC clearly has a role in setting the positions of all nucleosomes relative to the TSS on all genes, but heavily transcribed genes might be associated with more RSC because their chromatin is repeatedly disrupted.

Our observation that the NDRs of weakly transcribed genes are most affected by RSC depletion might reflect an indirect effect of RSC because RSC is not enriched at their NDRs. However, RSC is enriched at some promoters ( $\mathrm{Ng}$ et al. 2002; Floer et al. 2010), suggesting that it has a complex role in organizing chromatin. Nevertheless, whether direct or indirect, the effects of RSC depletion are truly global; our heat map and nucleosome shift analyses indicate that relatively few promoters are unaffected.

In contrast, none of the global effects observed in RSCdepleted cells are observed in the absence of SWI/SNF, a closely related remodeling complex, indicating that specific properties of RSC have been identified. SWI/SNF might act primarily on inducible genes (Peterson and Tamkun 1995; Tolkunov et al. 2011) such that its contribution is not apparent without induction. Indeed, SWI/SNF has a particular role in organizing centromeric chromatin (Gkikopoulos et al. 2011a). Our data are consistent with those of Wippo et al. (2011), showing that SWI/SNF cannot substitute for RSC to position nucleosomes correctly in vitro. A more restricted role for SWI/SNF in gene regulation is also suggested by the much lower abundance of SWI/SNF relative to RSC in yeast (Ghaemmaghami et al. 2003).

Nucleosome phasing can be understood in terms of a barrier model (Kornberg 1981; Mavrich et al. 2008; Chereji and Morozov 2011; Chereji et al. 2011; Rube and Song 2014), in which proteins bound to specific promoter DNA sequences sterically dictate the assembly of nucleosomes on both sides, resulting in phasing with respect to the DNA sequence. Thus, it is the boundaries of the phasing barrier that define the phasing origins (Fig. 9A). Given that the TSS is actually located within the +1 nucleosome, the putative phasing barrier cannot be located at the TSS itself. Instead, we argue that the phasing barrier is located at a fixed distance upstream of the TSS, within the NDR, probably adjacent to the +1 nucleosome. Similarly, upstream phasing begins at the upstream boundary of the barrier, which determines the location of the -1 nucleosome. Thus, the NDR might represent the footprint of the phasing barrier.

It is interesting that the NDR often observed at the 3 '-ends of genes apparently does not act as a barrier: The 3 '-end (TES) has only weak phasing properties and the trough corresponding to the $3^{\prime}$-NDR is much shallower than that of the $5^{\prime}$-NDR (Supplemental Fig. S4A; Mavrich et al. 2008). This suggests that $3^{\prime}$-barriers are much less prevalent than $5^{\prime}$-barriers and would explain why the $3^{\prime}$ region located between convergent genes does not block interference between arrays emanating from the flanking 5 '-barriers. In fact, TESs located between convergent genes have virtually no effect on phasing (Supplemental Fig. S4B).

The problem of what happens to nucleosomal arrays when the distance between NDRs is a nonintegral number of nucleosomal repeats has been addressed previously (Vaillant et al. 2010). Nucleosome occupancy data from a ChIP-on-chip study were used to divide yeast genes into three classes: (1) genes with "crystal" chromatin structure, which are organized into a single nucleosomal array; (2) genes with "bistable" chromatin, which are organized into one of two alternative arrays with different spacing, such that the first and last nucleosomes between two NDRs are the same in both arrays and one array has one more nucleosome than the

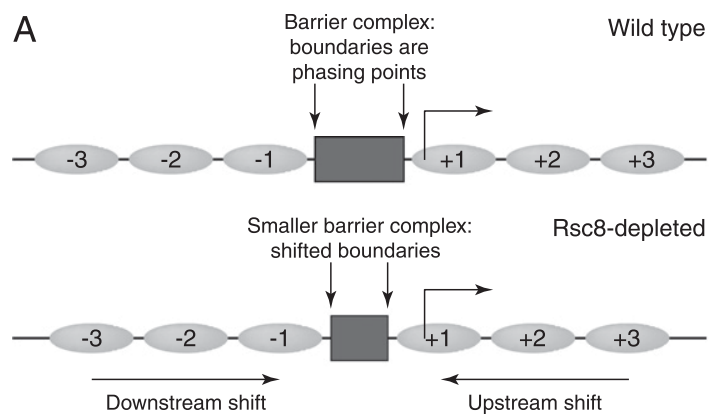

B Constructive interference: in phase arrays of nucleosomes
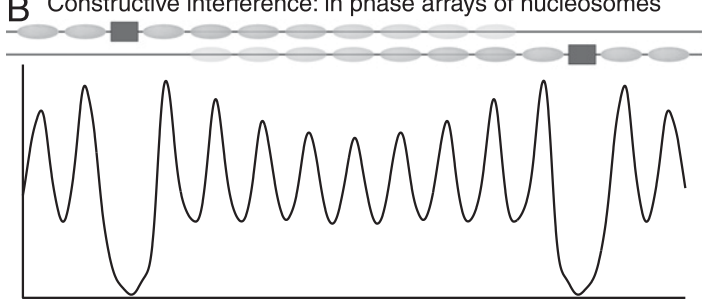

Destructive interference: out of phase arrays of nucleosomes

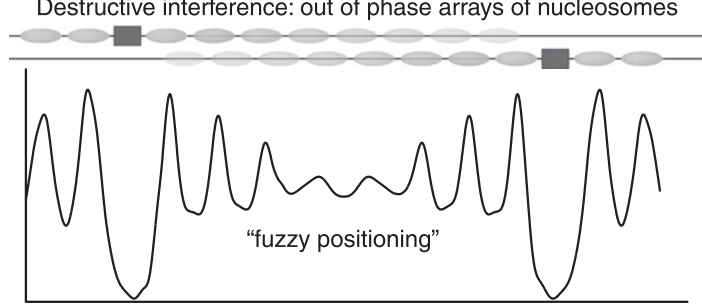

Figure 9. Bidirectional barrier model to account for phasing in wildtype and Rsc8-depleted cells. $(A)$ Nucleosome phasing is due to the presence of a putative sequence-specific barrier complex located in the NDR at the promoter, which acts through steric occlusion of nucleosomes. Its boundaries are the phasing points. The TSS is actually located within the +1 nucleosome. To explain its phasing properties, the TSS must be located at a fixed distance downstream from the barrier. In Rsc8-depleted cells, we propose that the barrier complex is smaller (reduced footprint) such that its boundaries direct phasing farther upstream of the TSS, accounting for narrowing of the NDR. Genes with filled promoters have no barrier and so phasing here will depend on the nearest barriers. $(B)$ Interference between arrays beginning at neighboring barriers will be constructive or destructive, depending on whether their separation is an integral number of nucleosomal repeats. Destructive interference will contribute to "fuzzy positioning."

other; (3) genes with a more complex chromatin structure (Supplemental Fig. S6A). This alternative spacing model predicts that bistable genes will display two or more sets of nucleosome phasing peaks, corresponding to the alternative arrays. However, our data do not support this attractive model: We see no evidence for arrays with altered spacing in any of our phasing analyses. Furthermore, analysis of our data using the lists of crystal and bistable genes from Vaillant et al. (2010) shows that they have identical phasing and nucleosome spacing (Supplemental Fig. S6B). The most likely reason for the discrepancy between our analysis and that of Vaillant et al. (2010) is that our nucleosome sequence data have much higher resolution than the microarray data used in their study.

Bidirectional phasing is the simple result of phasing from both borders of the barrier. The apparent loss of phasing power with distance from the phasing complex is the result of interference between the activities of neighboring barriers, the strength of which depends on the distance between the barriers (Fig. 9B). If the distance between their proximal borders is a near-integral 
number of nucleosomal repeats (165 bp), then arrays originating from both phasing points will coincide (constructive interference), and a regular nucleosomal pattern will be observed between the two barriers. If, on the other hand, the distance between the phasing points is a nonintegral number of nucleosomal repeats, then nucleosomes in arrays originating from these points will occupy different positions, resulting in destructive interference. In this case, nucleosomes close to the phasing points are more regularly positioned than those in between. Thus, "fuzzy" positioning can be explained in part by alternative arrays emanating from neighboring phasing points.

A reasonable biochemical mechanism for nucleosome phasing interference is that the ATP-dependent chromatin remodeling factors CHD1 and ISW1 (Gkikopoulos et al. 2011b; Zhang et al. 2011) are recruited to promoters, perhaps by barrier complexes, and then translocate away from the phasing points in both directions, spacing the nucleosomes as they proceed. Thus, remodelers recruited at neighboring phasing points may travel toward one another, perhaps colliding. The nucleosomes on the DNA between two barriers would be subjected to repositioning every time a remodeler passes along the DNA in the opposite direction, unless the distance between the phasing points is $165 n$, in which special case, the same positions would be utilized by remodelers coming from both directions (constructive interference). Such regions might fold into more regular higher order chromatin structures.

According to our model, the barriers at promoters in Rsc8depleted cells are different from those in wild-type cells. At promoters where narrowing of the NDR is observed due to invasion by the +1 and -1 nucleosomes, the phasing points have shifted farther into the NDR. This can be explained if the footprint of the barrier complex is smaller in Rsc8-depleted cells (Fig. 9A). At the few promoters where nucleosome fill-in occurs (e.g., gene clusters 6 and 9 in Fig. 4B), the barrier may have been eliminated. In this case, phasing interference will occur between the nearest promoters occupied by barrier complexes. At promoters with large intergenic regions, the situation is more complicated, with multiple complexes possible at different locations, predicting multiple upstream phasing points, depending on how stable they are.

The nature of the barrier complexes is an open question. The barrier model requires that the barrier is present at most promoters most of the time in most cells, suggesting that a relatively stable complex is involved. In principle, any complex with a footprint of the appropriate size within the NDR could act as a barrier. A reasonable possibility is that the barrier includes RSC itself, or a RSCnucleosome complex, the DNA in which might be partially unwound and therefore shorter than a canonical nucleosome (Floer et al. 2010). The latter fits with our observation that the barrier complex is somewhat smaller than a canonical nucleosome in wild-type cells and much smaller in Rsc8-depleted cells, although such nucleosomes would have to be relatively accessible to MNase to account for the NDR. There is evidence for a "fragile" nucleosome formed at some promoters, so-called because it is unusually sensitive to MNase digestion (Weiner et al. 2010; Xi et al. 2011), although its composition has not been established.

An alternative possibility is that the barrier is a transcription complex, not a nucleosome. A clear example is the stable TFIIIBTFIIIC transcription complex formed on tRNA genes, which is about the same size as a nucleosome and phases nucleosomes bidirectionally (Nagarajavel et al. 2013), and is regulated by RSC (Ng et al. 2002; Parnell et al. 2008). The equivalent complex for genes transcribed by RNA polymerase II is the pre-initiation complex, which is formed at the TATA box (or TATA-like elements) within the NDR and is located at a fixed distance from the TSS (Rhee and Pugh 2012; Struhl and Segal 2013). It may be significant that transcription is heavily reduced in Rsc8-depleted cells (Parnell et al. 2008; Hartley and Madhani 2009), perhaps suggesting the loss of critical components of a transcription complex, reflected by the smaller footprint. Another possibility is that sequence-specific transcription factors are responsible for phasing, although these typically have very short residence times, and stability is likely to be important in maintaining the NDR. If sequence-specific transcription factors play a direct role, they could be components of a stable complex. In fact, this is true of RSC itself, since the Rsc 3 and Rsc30 subunits bind specifically to DNA (Angus-Hill et al. 2001; Badis et al. 2008).

In summary, our observations demonstrate that the RSC complex plays a central role in defining the chromatin structure of yeast: In the absence of RSC, the NDR becomes narrower because the barrier complex is smaller, resulting in repositioning of the +1 and -1 nucleosomes next to it, and then the more distal nucleosomes are repositioned to maintain the correct spacing, resulting in a global shift of nucleosomes with respect to genes, and new phasing interference patterns.

\section{Methods}

\section{Yeast strains}

MCY3860 (MAT $\alpha$ swh341::HIS3 his34200 lys2-801 ura3-52 pGAL::SWH3-URA3) and MCY3647 (MATa his34200 lys2-801 leu2-3,112 ura3-52) were grown in synthetic complete (SC) medium containing $2 \%$ galactose and then switched to glucose as described (Treich and Carlson 1997). Rsc8/Swh3-depleted cells were harvested when their growth reached a plateau; wild-type cells were harvested in mid-log phase. YDC111 (MATa ade2-1 can1-100 leu2-3, 112 trp1-1 ura3-1 RAD5+) and YDC188 (MATa ade2-1 can1-100 leu2-3,112 trp1-1 ura3-1 RAD5+ ISW1-FL3-KanMX snf2L::URA3) (Kim et al. 2006) were grown in SC medium with $2 \%$ glucose to mid-log phase.

\section{Nucleosome sequencing}

Nuclei were prepared and digested to nucleosome core particles with MNase as described previously (Cole et al. 2012a), except that gel-purified core particle DNA was ligated to Index PE adapters (Illumina) and amplified with Illumina Index primers using 15 cycles of PCR. Two biological replicate experiments were performed. The RSC-depletion experiments yielded three data sets (core particle DNA from two slightly different levels of MNase digestion was sequenced in the second experiment) with 50-nt paired reads: MCY3647 gave 23,068,963, 11,156,905, and 8,416,401 aligned paired reads; MCY3860 gave 16,009,668, 11,154,495, and $10,856,926$ aligned paired reads. The $\operatorname{snf} 2 \Delta$ analysis was performed as described previously (Cole et al. 2011a): YDC188 yielded $18,565,840$ and $17,101,226$ aligned paired reads of 36 or $60 \mathrm{nt}$, respectively. The snf $2 \Delta$ data were compared with our published data for YDC111 (Cole et al. 2011a), because YDC188 was derived from this wild-type W303 strain (the MCY strains are S288C). However, the wild-type strains were indistinguishable in the analyses described here.

\section{Bioinformatic analysis}

Paired reads were aligned to the Saccharomyces cerevisiae genome (sacCer2) using Bowtie 2 (Langmead and Salzberg 2012). MATLAB 
scripts to determine average occupancy and midpoint phasing relative to the TSS are provided (Supplemental Material). For each data set, nucleosome sequences in the range 140-160 bp were selected and the locations of their dyads were inferred by calculating the midpoint coordinate. Combined TSS and TES data were obtained from three sources (Nagalakshmi et al. 2008; Arribere and Gilbert 2013; Park et al. 2014). A mathematical description of the active nucleosome phasing model is provided in Supplemental Methods.

\section{Data access}

The sequencing data from this study have been submitted to the NCBI Gene Expression Omnibus (GEO; http://www.ncbi.nlm.nih. gov/geo/) under accession number GSE49512.

\section{Acknowledgments}

This research was supported by the Intramural Research Program of the NIH (NICHD). We thank Gary Felsenfeld, Tara Burke, Peter Eriksson, and Josefina Ocampo for helpful comments on the manuscript. We thank the Tufts University Core Facility for pairedend sequencing of the snf $2 \Delta$ strain and the NHLBI Core Facility (Yoshi Wakabayashi, Yan Luo, Ting Ni, and Jun Zhu) for paired-end sequencing of the RSC strains. We thank Marian Carlson for strains MCY3860 and MCY3647.

\section{References}

Angus-Hill ML, Schlichter A, Roberts D, Erdjument-Bromage H, Tempst P, Cairns BR. 2001. A Rsc3/Rsc30 zinc cluster dimer reveals novel roles for the chromatin remodeler RSC in gene expression and cell cycle control. Mol Cell 7: 741-751.

Arribere JA, Gilbert WV. 2013. Roles for transcript leaders in translation and mRNA decay revealed by transcript leader sequencing. Genome Res 23: 977-987.

Badis G, Chan ET, van Bakel H, Pena-Castillo L, Tillo D, Tsui K, Carlson CD, Gossett AJ, Hasinoff MJ, Warren CL, et al. 2008. A library of yeast transcription factor motifs reveals a widespread function for Rsc3 in targeting nucleosome exclusion at promoters. Mol Cell 32: 878-887.

Blank TA, Becker PB. 1995. Electrostatic mechanism of nucleosome spacing. J Mol Biol 252: 305-313.

Cairns BR, Lorch Y, Li Y, Zhang M, Lacomis L, Erdjument-Bromage H, Tempst P, Du J, Laurent B, Kornberg RD. 1996. RSC, an essential, abundant chromatin remodeling complex. Cell 87: 1249-1260.

Cairns BR, Schlichter A, Erdjument-Bromage H, Tempst P, Kornberg RD, Winston F. 1999. Two functionally distinct forms of the RSC nucleosome remodeling complex, containing essential AT hook, BAH and bromodomains. Mol Cell 4: 715-723.

Chereji RV, Morozov AV. 2011. Statistical mechanics of nucleosomes constrained by higher order structure. J Stat Phys 144: 379-404.

Chereji RV, Tolkunov D, Locke G, Morozov AV. 2011. Statistical mechanics of nucleosome ordering by chomatin-structure-induced two-body interactions. Phys Rev E Stat Nonlin Soft Matter Phys 83: 050903.

Clapier CR, Cairns BR. 2009. The biology of chromatin remodeling complexes. Аnnu Rev Biochem 78: 273-304.

Cole HA, Howard BH, Clark DJ. 2011a. Activation-induced disruption of nucleosome position clusters on the coding regions of Gcn4-dependen genes extends into neighbouring genes. Nucleic Acids Res 39: 95219535.

Cole HA, Howard BH, Clark DJ. 2011b. The centromeric nucleosome of budding yeast is perfectly positioned and covers the entire centromere. Proc Natl Acad Sci 108: 12687-12692.

Cole HA, Howard BH, Clark DJ. 2012a. Genome-wide mapping of nucleosomes in yeast using paired-end sequencing. Methods Enzymol 513: $145-168$.

Cole HA, Nagarajavel V, Clark DJ. 2012b. Perfect and imperfect nucleosome positioning in yeast. Biochim Biophys Acta 1819: 639-643.

Cui F, Cole HA, Clark DJ, Zhurkin VB. 2012. Transcriptional activation of yeast genes disrupts intragenic nucleosome phasing. Nucleic Acids Res 40: $10753-10764$.

Da G, Lenkart J, Zhao K, Shiekhattar R, Cairns BR, Marmorstein R. 2006. Structure and function of the SWIRM domain, a conserved protein module found in chromatin regulatory complexes. Proc Natl Acad Sci 103: $2057-2062$.
Damelin M, Simon I, Moy TI, Wilson B, Komili S, Tempst P, Roth FP, Young RA, Cairns BR, Silva PA. 2002. The genome-wide localization of Rsc9, a component of the RSC chromatin-remodeling complex, changes in response to stress. Mol Cell 9: 563-573.

Elfving N, Chereji RV, Bharatula V, Björklund S, Morozov AV, Broach JR. 2014. A dynamic interplay of nucleosome and Msn2 binding regulates kinetics of gene activation and repression following stress. Nucleic Acids Res 42: 5468-5482.

Floer M, Wang X, Prabhu V, Berrozpe G, Narayan S, Spagna D, Alvarez D, Kendall J, Krasnitz A, Stepansky A, et al. 2010. A RSC/nucleosome complex determines chromatin architecture and facilitates activator binding. Cell 141: 407-418.

Ghaemmaghami S, Huh W, Bower K, Howson RW, Belle A, Dephoure N, O'Shea EK, Weissman JS. 2003. Global analysis of protein expression in yeast. Nature 425: 737-741.

Gkikopoulos T, Singh V, Tsui K, Awad S, Renshaw MJ, Scholfield P, Barton GJ, Nislow C, Tanaka TU, Owen-Hughes T. 2011a. The SWI/SNF complex acts to constrain distribution of the centromeric histone variant Cse4. EMBO J 30: 1919-1927.

Gkikopoulos T, Schofield P, Singh V, Pinskaya M, Mellor J, Smolle M, Workman JL, Barton GJ, Owen-Hughes T. 2011b. A role for Snf2-related nucleosome-spacing enzymes in genome-wide nucleosome organization. Science 333: 1758-1760.

Hartley PD, Madhani HD. 2009. Mechanisms that specify promoter nucleosome location and identity. Cell 137: 445-458.

Holstege FC, Jennings EG, Wyrick JJ, Lee TI, Hengartner CJ, Green MR, Golub TR, Lander ES, Young RA. 1998. Dissecting the regulatory circuitry of a eukaryotic genome. Cell 95: 717-728.

Hota SK, Bartholomew B. 2011. Diversity of operation in ATP-dependent chromatin remodelers. Biochim Biophys Acta 1809: 476-487.

Ioshikhes IP, Albert I, Zanton SJ, Pugh BF. 2006. Nucleosome positions predicted through comparative genomics. Nat Genet 38: 1210-1215.

Ito T, Bulger M, Pazin MJ, Kobayashi R, Kadonaga JT. 1997. ACF, an ISWIcontaining and ATP-utilizing chromatin assembly and remodeling factor. Cell 90: 145-155.

Kim Y, McLaughlin N, Lindstrom K, Tsukiyama T, Clark DJ. 2006. Activation of Saccharomyces cerevisiae HIS3 results in Gcn4-dependent, SWI/SNFdependent mobilization of nucleosomes over the entire gene. Mol Cell Biol 26: 8607-8622.

Kornberg RD. 1981. The location of nucleosomes in chromatin: specific or statistical? Nature 292: 579-580.

Langmead B, Salzberg SL. 2012. Fast gapped-read alignment with Bowtie 2. Nat Methods 9: 357-359.

Lee CK, Shibata Y, Rao B, Strahl BD, Lieb JD. 2004. Evidence for nucleosome depletion at active regulatory regions genome-wide. Nat Genet 36: 900905.

Lee W, Tillo D, Bray N, Morse RH, Davis RW, Hughes TR, Nislow C. 2007. A high-resolution atlas of nucleosome occupancy in yeast. Nat Genet 39: 1235-1244.

Lohr D, Kovacic RT, van Holde KE. 1977. Quantitative analysis of the digestion of yeast chromatin by staphylococcal nuclease. Biochem 16: 463-471.

Lorch Y, Zhang M, Kornberg RD. 2001. RSC unravels the nucleosome. Mol Cell 7: 89-95.

Luger K, Mäder AW, Richmond RK, Sargent DF, Richmond TJ. 1997. Crystal structure of the nucleosome core particle at 2.8 A resolution. Nature 389: 251-260.

Mavrich TN, Ioshikhes IP, Venters BJ, Jiang C, Tomsho LP, Qi J, Schuster SC, Albert I, Pugh BF. 2008. A barrier nucleosome model for statistical positioning of nucleosomes throughout the yeast genome. Genome Res 18: $1073-1083$.

Nagalakshmi U, Wang Z, Waern K, Shou C, Raha D, Gerstein M, Snyder M. 2008. The transcriptional landscape of the yeast genome defined by RNA sequencing. Science 320: 1344-1349.

Nagarajavel V, Iben JR, Howard BH, Maraia RJ, Clark DJ. 2013. Global 'bootprinting' reveals the elastic architecture of the yeast TFIIIB-TFIIIC transcription complex in vivo. Nucleic Acids Res 41: 8135-8143.

Narlikar GJ, Sundaramoorthy R, Owen-Hughes T. 2013. Mechanisms and functions of ATP-dependent chromatin-remodeling enzymes. Cell 154: 490-503.

$\mathrm{Ng} \mathrm{HH}$, Robert F, Young RA, Struhl K. 2002. Genome-wide location and regulated recruitment of the RSC nucleosome remodeling complex. Genes Dev 16: 806-819.

Park D, Morris AR, Battenhouse A, Iyer VR. 2014. Simultaneous mapping of transcript ends at single-nucleotide resolution and identification of widespread promoter-associated non-coding RNA governed by TATA elements. Nucleic Acids Res doi: 10.1093/nar/gkt1366.

Parnell TJ, Huff JT, Cairns BR. 2008. RSC regulates nucleosome positioning at Pol II genes and density at Pol III genes. EMBO J 27: 100-110.

Peterson CL, Tamkun JW. 1995. The SWI-SNF complex: a chromatin remodeling machine? Trends Biochem Sci 20: 143-146. 
Rhee HS, Pugh BF. 2012. Genome-wide structure and organisation of eukaryotic pre-initiation complexes. Nature 483: 295-301.

Rube HT, Song JS. 2014. Quantifying the role of steric constraints in nucleosome positioning. Nucleic Acids Res 42: 2147-2158.

Saha A, Wittmeyer J, Cairns BR. 2002. Chromatin remodeling by RSC involves ATP-dependent DNA translocation. Genes Dev 16: 2120-2134.

Shukla MS, Syed SH, Montel F, Faivre-Moskalenko C, Bednar J, Travers A, Angelov D, Dimitrov S. 2010. Remosomes: RSC-generated nonmobilized particles with approximately 180 bp DNA loosely associated with the histone octamer. Proc Natl Acad Sci 107: 1936-1941.

Struhl K, Segal E. 2013. Determinants of nucleosome positioning. Nat Struct Mol Biol 20: 267-273.

Thomas JO, Furber V. 1976. Yeast chromatin structure. FEBS Lett 66: 274-280.

Tolkunov D, Zawadzki KA, Singer C, Elfving N, Morozov AV, Broach JR. 2011. Chromatin remodelers clear nucleosomes from intrinsically unfavorable sites to establish nucleosome-depleted regions at promoters. Mol Biol Cell 22: 2106-2118.

Treich I, Carlson M. 1997. Interaction of a Swi3 homologue with Sth1 provides evidence for a Swi/Snf-related complex with an essential function in Saccharomyces cerevisiae. Mol Cell Biol 17: 1768-1775.

Tsukiyama T, Palmer J, Landel CC, Shiloach J, Wu C. 1999. Characterization of the Imitation Switch subfamily of ATP-dependent chromatin remodeling factors in Saccharomyces cerevisiae. Genes Dev 13: $686-697$.

Vaillant C, Palmeira L, Chevereau G, Audit B, d'Aubenton-Carafa C, Thermes C, Arneodo A. 2010. A novel strategy of transcription regulation by intragenic nucleosome ordering. Genome Res 20: 59-67. van Holde KE. 1988. Chromatin. Springer-Verlag, New York.

Weiner A, Hughes A, Yassour M, Rando OJ, Friedman N. 2010. Highresolution nucleosome mapping reveals transcription-dependent promoter packaging. Genome Res 20: 90-100.

Wippo CJ, Israel L, Watanabe S, Hochheimer A, Peterson CL, Korber P. 2011. The RSC chromatin remodelling enzyme has a unique role in directing the accurate positioning of nucleosomes. EMBO J 30: 1277-1288.

Xi Y, Yao J, Chen R, Li W, He X. 2011. Nucleosome fragility reveals novel functional states of chromatin and poises genes for activation. Genome Res 21: 718-724.

Yen K, Vinayachandran V, Batta K, Koerber RT, Pugh BF. 2012. Genome-wide nucleosome specificity and directionality of chromatin remodelers. Cell 149: 1461-1473.

Yuan G, Liu Y, Dion MF, Slack MD, Wu LF, Altschuler SJ, Rando OJ. 2005. Genome-scale identification of nucleosome positions in Saccharomyces cerevisiae. Science 309: 626-630.

Zawadzki KA, Morozov AV, Broach JR. 2009. Chromatin-dependent transcription factor accessibility rather than nucleosome remodeling predominates during global transcriptional restructuring in Saccharomyces cerevisiae. Mol Biol Cell 20: 3503-3513.

Zhang Z, Wippo CJ, Wal M, Ward E, Korber P, Pugh BF. 2011. A packing mechanism for nucleosome organization reconstituted across a eukaryotic genome. Science 332: 977-980.

Received April 9, 2014; accepted in revised form July 8, 2014. 


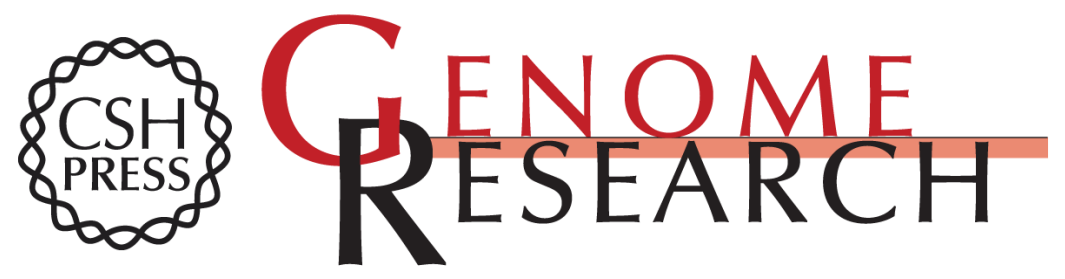

\section{RSC-dependent constructive and destructive interference between opposing arrays of phased nucleosomes in yeast}

Dwaipayan Ganguli, Razvan V. Chereji, James R. Iben, et al.

Genome Res. 2014 24: 1637-1649 originally published online July 11, 2014

Access the most recent version at doi:10.1101/gr.177014.114

Supplemental Material

References

Creative

Commons

License

Email Alerting Service
http://genome.cshlp.org/content/suppl/2014/08/01/gr.177014.114.DC1

This article cites 59 articles, 22 of which can be accessed free at: http://genome.cshlp.org/content/24/10/1637.full.html\#ref-list-1

This article is distributed exclusively by Cold Spring Harbor Laboratory Press for the first six months after the full-issue publication date (see

$\mathrm{http}: / / g$ enome.cshlp.org/site/misc/terms.xhtml). After six months, it is available under a Creative Commons License (Attribution-NonCommercial 4.0 International), as described at http://creativecommons.org/licenses/by-nc/4.0/.

Receive free email alerts when new articles cite this article - sign up in the box at the top right corner of the article or click here.

\section{Affordable, Accurate Sequencing.}

To subscribe to Genome Research go to:

https://genome.cshlp.org/subscriptions 